\title{
Flow curve prediction of cold forging steel by artificial neural network model
}

\author{
Fatih Kocatürk, M. Burak Toparli, Barıș Tanrıkulu, Sezgin Yurtdaş, Doğuş Zeren and Cenk Kılıçaslan
}

Fatih Kocatürk. R\&D Center, Norm Civata San. ve Tic. A.Ş., AOSB, İzmir, Turkey

Graduate School, Applied Mathematics and Statistics, İzmir University of Economics, İzmir, Turkey

Corresponding author: fatih.kocaturk@norm-fasteners.com.tr

M. Burak Toparli. R\&D Center, Norm Civata San. ve Tic. A.Ş., AOSB, İzmir, Turkey

Barıș Tanrıkulu. R\&D Center, Norm Cıvata San. ve Tic. A.Ş., AOSB, İzmir, Turkey

The Graduate School of Natural and Applied Sciences, Dokuz Eylül University, İzmir, Turkey

Sezgin Yurtdaş. R\&D Center, Norm Civata San. ve Tic. A.Ş., AOSB, İzmir, Turkey

Mechanical Engineering Department, Katip Çelebi University, İzmir, Turkey

Doğuş Zeren. R\&D Center, Norm Civata San. ve Tic. A.Ş., AOSB, İzmir, Turkey

Cenk Kılıçaslan. R\&D Center, Norm Cıvata San. ve Tic. A.Ş., AOSB, İzmir, Turkey

\begin{abstract}
A limited number of material models or flow curves are available in commercial finite element softwares at varying temperature and strain rate ranges for plasticity analysis. To obtain more realistic finite element results, flow curves at wide temperature and strain rate ranges are required. For this purpose, a material model for a medium carbon alloy steel material which is used for fastener production was prepared. Firstly, flow curves of the material were obtained at 4 temperatures $\left(20,100,200,400{ }^{\circ} \mathrm{C}\right)$ and 3 strain rates $(1$, $10,50 \mathrm{~s}^{-1}$ ). Then, experimental data was used to construct an artificial neural networks model (ANN) for the material. $75 \%$ of the experimental data was used to train the model and the rest was employed for validation and verification. ANN model used in flow curve prediction was developed using the scikit-learn library on Python. Temperature, strain rate and strain were employed as input parameters and flow stress as output parameter in ANN model. In order to increase the accuracy of the ANN model, the number of hidden layers and the number of neurons were also optimized by mean squared error approach. As a result of studies, an ANNbased material model that can be used for wide range of temperature and strain rate values were developed based on the experimental data.
\end{abstract}

Keywords. Flow Curve Prediction, Artificial Neural Network, Python, Medium Carbon Alloy Steel

\section{Introduction}

Numerical simulation softwares are actively used in many areas of the industry today. Boundary conditions and material properties must be defined as accurately as possible in order to coincide the results of simulation studies with actual production conditions. In this context, this study was carried out to expand the material models with the help of Artificial Neural Network (ANN) in order to increase the analysis capability of the Simufact.forming software, which is actively used for bolt forming and tool analysis. Since there is a limited number of material data in the Simufact.forming software, the software creates a value outside the data range by interpolating during the analysis phase. In order to prevent interpolating and to obtain more accurate analysis results, ANN models were used to reproduce the existing material data and were integrated into the simulation software. The artificial neural network models can be adapted to solve an optimization problem by appropriately selecting synaptic connections. In the study conducted by Dhingra and Rao (1992) [1], neural network models were designed to solve four design optimization problems and the model results were presented to show the computational power of these networks. Optimum solutions obtained by using neural networks are well adapted to the solutions obtained using gradient-based search techniques. Garrett et al. (1993) [2] presented the application of neural network technology to several engineering problems (problems requiring adaptive control, problems requiring a mapping or classification of distributed noisy data, problems requiring the simultaneous solution of many different constraints for multi-objective functions). The implemented applications have demonstrated the potential of neural networks to solve certain types of engineering problems. In master thesis 
Flow curve prediction of cold forging steel by artificial neural network model

of Denizer (2008) [3], ANN was used to draw the constitutive laws of tungsten fibre reinforced bulk metallic glass matrix composites. The purpose of the inverse analysis performed through ANN was to optimize the values of Voce and power law parameters and a total of seven parameters including the freezing temperature. First, a feed forward ANN was created that tried to match the FEA predictions run multiple times by random selection of seven parameters, then inverse ANN models were created to optimize the values of the seven parameters. Finally, optimized parameters were entered into forward ANN and compared with experimental data. The ANN approach was proposed by Toros and Öztürk (2011) [4] to determine the material flow curves of strain-hardened 5083-H111 and 5754-0 Al-Mg alloys between room temperature and $300^{\circ} \mathrm{C}$ and $0.0016-0.16 \mathrm{~s}^{-1}$ strain rate range. Tensile tests were conducted to determine the material response at various temperatures and strain rates, and the ANN model was developed to estimate the flow curves of materials using experimental data. A three-layer feed forward network is trained with the BFGS (Broyden, Fletcher, Goldfarb, and Shanno) algorithm. The results revealed that the values predicted by the ANN model were in good agreement with the experimental data. Various applications of soft computing (SC) tools in metal forming processes were reviewed by Pattanaik (2013) [5]. In this context, they studied ANN, fuzzy logic, genetic algorithms and the hybridization of these methods to show the ability of SC tools and techniques to effectively address various metal forming problems and related issues. They observed that the basic applications of the mentioned SC tools can be classified into the areas of design, optimization and forming processes prediction. Metal forming processes using SC tools were: Design of dies and preforms, rolling force/torque estimation, optimum force calculation, springback prediction and many more applications during forging, rolling, bending, extrusion and different sheet metal works. Mehtedi et al. (2019) [6] used the ANN model to estimate the flow curves of ZAM100 magnesium alloy sheets under hot forging conditions and obtained predictions with low error rates. A computer-aided tool in order to estimate the physical and mechanical properties used in the determination of metallic materials was developed by Merayo et al. (2020) [7]. The developed system is based on the use of the ANN model, supported by the collection of large data on the properties of thousands of materials. After training the ANN model, the system was able to make predictions about the material density and Young's modulus with an average confidence of 99\% and more than 98\%, respectively. A semi-autonomous vision-based method for detecting loose bolts in critical joints was proposed by Huynh et al. (2019) [8]. The method was using the idea of estimating the rotation angle of bolts from connection images by integrating deep learning technology with image processing techniques. For this case, a regional convolutional neural network based deep learning algorithm was developed to automatically detect and crop the reasonable bolts in the connection image, and the results showed that the perspective angle should not exceed 40 degrees for the accuracy of detection results. Zhang et al. (2020) [9] aimed to validate the use of machine vision and deep learning for structural health monitoring, focusing on the practice of detecting a specific bolt loosening. In this direction, a data set containing 300 images was collected first and then a faster region-based convolutional neural network was trained and validated. Test results showed that bolt damage detection was at an average sensitivity rate of 0.9503 .

Obtaining flow curves of materials at different temperature, strain rate and plastic strain values is costly due to existing test systems. Studies in the literature where material flow curves are predicted with ANN models verify the ability of neural networks to predict material flow curves. The aim of this study is to establish an ANN model with reference to the material flow curves obtained by experimental methods and to reproduce the material flow curves for different temperature and strain rate values with the established model. Material flow curves for all temperature and strain rate ranges were obtained by utilising the constructed ANN model. Therefore, the errors are avoided resulting from interpolation or extrapolation that the simulation program uses for missing material data.

\section{Experimental Studies and Artificial Neural Network Model}

\subsection{Material}

In order to determine the flow curves of a medium carbon alloy steel raw material used in bolt production by cold 
forging method, cylindrical compression testing specimens having $8 \mathrm{~mm}$ diameter and $12 \mathrm{~mm}$ length measures were prepared in accordance with ASTM E9 standard [10]. Compression tests were carried out on the prepared cylindrical samples in ZWICK universal tensile/compression test device. The deformation rate was determined as $5 \mathrm{~mm} / \mathrm{min}$. corresponding to the quasi-static strain rate. Machine compression tables were lubricated with $\mathrm{MoS}_{2}$ based lubricant before each compression test. In this way, the friction condition (barrelling) affecting the material flow behaviour is minimized. Strain values were measured with load cell during compression test. In Fig. 1, the flow stress-plastic strain graphs for strain rate $1 \mathrm{~s}^{-1}$ and for different temperatures were given for a medium carbon alloy steel material. The flow stress-plastic strain graphs for $100{ }^{\circ} \mathrm{C}$ and three strain rates were given in Fig. 2. As can be seen in Fig. 1 and Fig. 2 , true stress values depending on the plastic strain increased as the temperature decreased and the strain rate value increased. The flow curves were obtained as a result of compression tests performed at four different temperatures $\left(20,100,200\right.$ and $\left.400{ }^{\circ} \mathrm{C}\right)$ and three different strain rates $\left(1,10,50 \mathrm{~s}^{-1}\right)$. Three tests were conducted for each temperature and strain rate values. By using data processing method, average flow curves for each temperature were found and final stress-strain curves of the materials were obtained. Within the scope of this study, an artificial neural network model was created for the material model, which is the most preferred in numerical simulation studies in cold forging industry. The flow curve data obtained by the experimental method were used in training and validation of the ANN model.

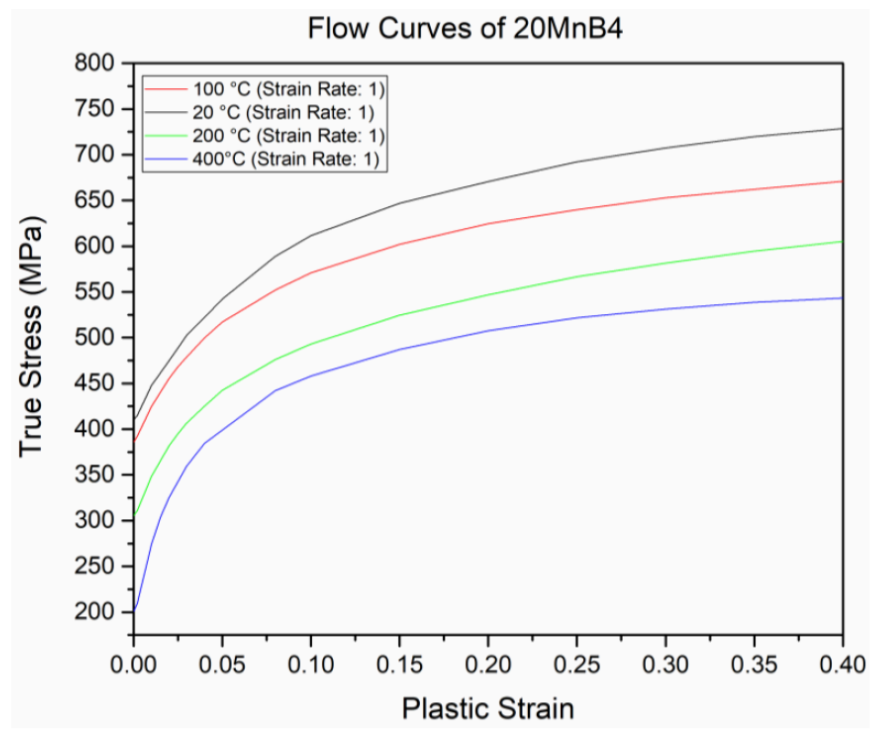

Fig. 1. Experimental flow curves for a medium carbon alloy steel material at different temperatures for strain rate 1 $\mathrm{s}^{-1}$. 
Flow curve prediction of cold forging steel by artificial neural network model

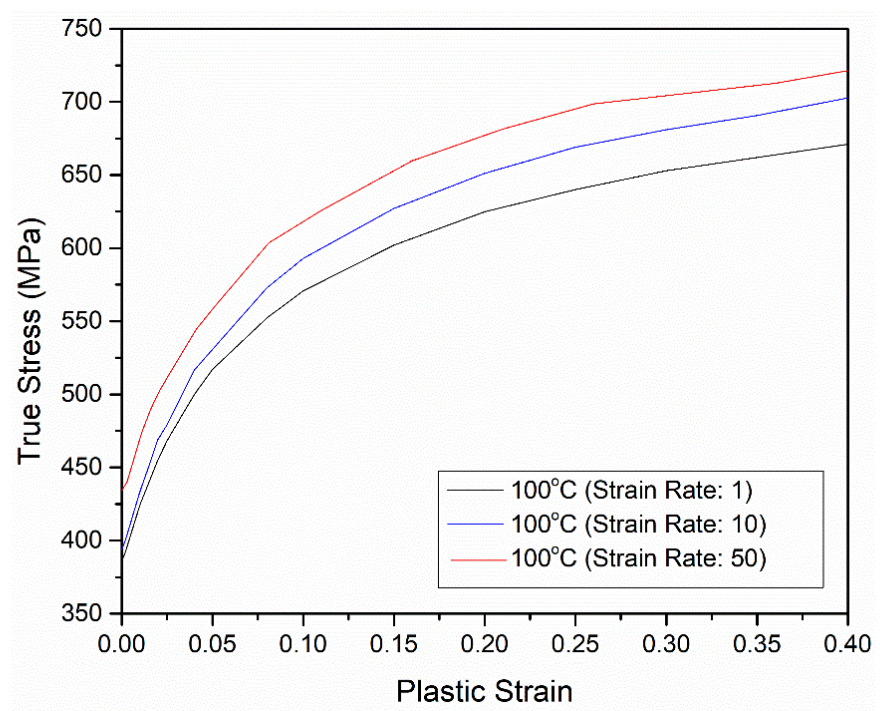

Fig. 2. Experimental flow curves for a medium carbon alloy steel material at different strain rates for $100^{\circ} \mathrm{C}$.

\subsection{ANN Model}

ANN model uses a parallel distributed computing paradigm as an alternative to sequential command-based computing. ANN is inspired by the structure of real neural networks in the human brain, and the knowledge gained from neuroscience is used to develop reliable, noise and error tolerant computing architectures that can make generalizations (Haykin (1999)[11]). ANN was defined as any computing architecture consisting of simple neural processors connected to each other massively parallel in Lau (1992) [12]. This definition shows that simple neural processors called neurons are central to the implementation of artificial neural networks. Neural network terminology architecture refers to the arrangement of neurons in layers and the pattern of synaptic connections between layers and between neurons. In a layered network, there is an input layer consisting of source nodes, an output layer of computation nodes, and one or more hidden neuron layers between input and output layers. There are two main classes of neural network architecture depending on the type of connection between neurons: i) Feed forward neural networks, ii) Recurrent neural networks. In feed forward neural networks, the signal flows in one direction between layers and there is no connection between neurons in the same layer. In recurrent neural networks there is at least one feedback loop that results in a dynamic system and can contain a unit delay operator where both types of connections are allowed.

A multilayer feed forward network or multilayer perceptron commonly mentioned in the literature consists of an input layer, one or more hidden layers, and an output layer. The training of a multilayer perceptron is performed in a controlled manner using the back propagation algorithm. At the beginning of the training process, random values are assigned to the synaptic weights of the network. The network is then presented with a sample of training at each iteration. The training sample consists of an input and a corresponding output. Training samples are obtained from experimental or simulation data. The network processes the input and generates an output, then it compares the output with the target. The difference between target and output determines the error. Then the synaptic weights of the network are changed by the learning algorithm in proportion to the error. The aim of the training process is to repeatedly reduce the error below a predetermined value. This requires the presentation of many training samples that make up a training set. A complete training set offering is called an epoch. This mode of supervised learning is called error correction learning. Back propagation algorithm (BPA) is based on error correction learning rule (McClelland et al. (1986)[13]). BPA consists of two stages: In the first stage, the input is presented and propagated through the 
network to calculate the output value for each unit (neuron). This output value gives an error signal for each output unit when compared to the targets. The second stage involves a backward transition over the network where the error signal is transmitted to each unit in the network and appropriate weight changes are made. Weight changes are made according to the steepest gradient descent method. Local slopes are calculated based on the location of a neuron in the network.

In order to predict flow curves of a medium carbon alloy steel material, the ANN model was created on Python programming language by using scikit-learn library which is an open access machine learning library running on Python. Multilayer Perceptron package in the scikit-learn library was used in order to construct ANN model. In order to construct the ANN, first of all, the input and output parameters must be determined. Taking into account the experimentally obtained material data given in Fig. 1, temperature, strain rate and plastic strain were determined as input parameters, and flow stress as output parameter. The values of input parameters were given in Table 1 . The model to be established with the specified input variables was designed to give the true stress or flow stress values for each combination of input values. The schema of constructed ANN model was given in Fig. 3.

Table 1. Input parameters and values used in the artificial neural network model.

\begin{tabular}{ll}
\hline Input parameters & Values \\
\hline Temperature $\left({ }^{\circ} \mathrm{C}\right)$ & $20,100,200,400$ \\
\hline Strain rate $(\mathrm{s}-1)$ & $1,10,50$ \\
\hline Plastic strain & $0-1$ \\
\hline
\end{tabular}

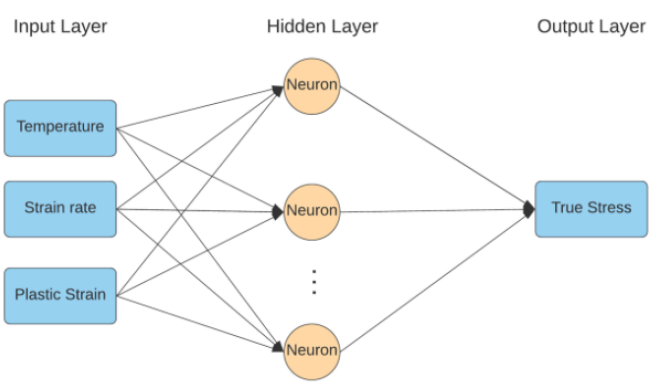

Fig. 3. The schema of constructed ANN model.

After obtaining the input data, the parameters affecting the performance of ANN model were determined and optimized for the best accuracy level of predictions. The parameters most affecting the prediction performance of the ANN model, which learns with the multilayer feed forward and back-propagation algorithm, are the activation function, solver algorithm, hidden layer size and the randomness value, respectively. Considering the number of input and output parameters, it was decided to construct the model with a single hidden layer. By increasing the number of hidden layers, deep learning structure is adopted and this model is generally used in cases where input and output parameters are more and their relationships are more complex. For this reason, the number of hidden layers was determined as one in this study. Since the output parameter is the true stress $(\sigma$ true $)$, which is a continuous variable, the hyperbolic tangent function, $\mathrm{f}(\mathrm{x})=\tanh (\mathrm{x})$, was determined as the activation function used for transition between hidden layers. Since the experimental data set is relatively small (300 rows), the BFGS algorithm, which is known to give better results in small data sets and is one of the quasi-Newton methods, was used as the solver algorithm. When a random 
value is required in software development, the random number generation library in the system is used. The random number generation function needs an initial (seed) value to start working. A different random number list is produced according to the entered initial value. This initial value is generally zero by default. By changing the initial value of the random number generation function, the algorithm result can be improved by using different random numbers within the algorithm, and obtaining the same result is guaranteed every time the algorithm is run. In the ANN model, it is important to find the random initial value that the ANN model gives the best result, since the weight values used in transitions between neurons are randomly assigned at first and random value assignments are used in the solver. For this purpose, the randomness value was examined together with the number of neurons. An optimization study was carried out for the randomness value. The randomness value was tested for all integers in the range $[0,100]$ and the best value was found that gives the lowest mean squared error (MSE). As a result of the study, the lowest MSE was obtained for the randomness value of 53. After determining the general structure of the model, the number of hidden layers and the solver algorithm, the process of obtaining the number of neurons to be defined for the hidden layer was started. At this stage, it was decided to use $75 \%$ of the experimentally obtained data for training the model and the remaining $25 \%$ for the validation of the model. Using the determined training data set, the process of determining the number of neurons to be used in the hidden layer was conducted. The number of neurons is one of the most important parameters to be determined for the ANN model. The number of neurons in the hidden layer can cause problems such as underfitting and overfitting. When the number of neurons is low, the model created does not fully reflect the data and may cause erroneous results. In case of high number of neurons, overfitting problem arises and the model is more affected by parameter changes. Therefore, a comprehensive study has been carried out for the number of neurons. MSE measure was also used in order to obtain the number of neurons. The change in the MSE parameter was recorded by increasing the number of neurons one by one starting from 2 neurons. The smallest number of neurons for which the MSE value is fixed was determined as the optimum number of neurons. As a result of the study conducted for the number of neurons, the graph given in Fig. 4 was obtained and the optimum number of neurons was determined as 9 . Finally, the parameters and their values used in the Multilayer Perceptron module were presented in Table 2.

Table 2. The parameters and their values used in the Multilayer Perceptron module.

\begin{tabular}{ll}
\hline Parameters & Values \\
\hline \# input parameters (Temperature, plastic strain, strain rate) & 3 \\
\hline \# output parameters (True stress) & 1 \\
\hline \# hidden layers & 1 \\
\hline \# neurons & 9 \\
\hline Activation function & tanh \\
\hline Solver & $1 \mathrm{bfgs}$ \\
\hline Randomness & 53 \\
\hline \# epochs & 1000 \\
\hline
\end{tabular}




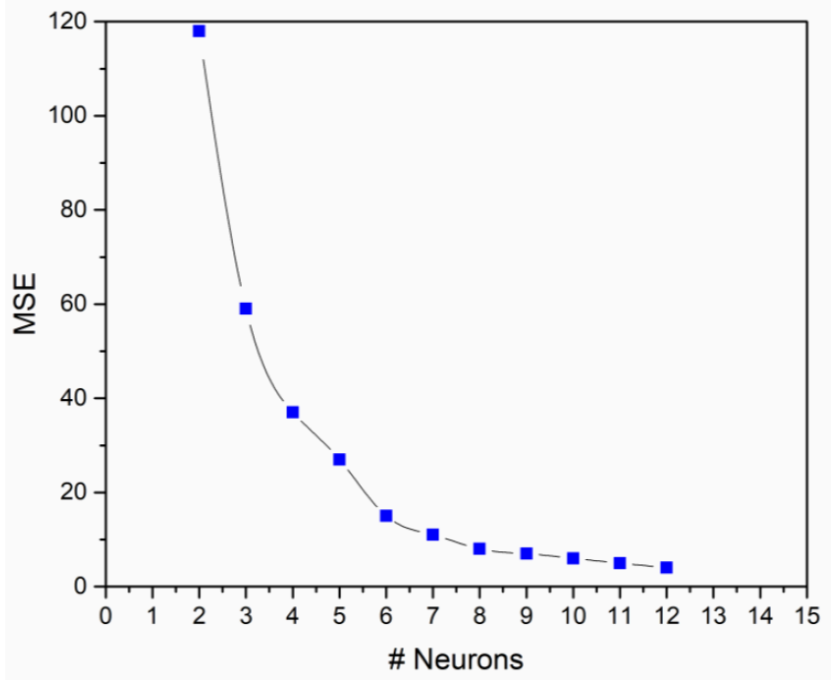

Fig. 4. MSE plot obtained to find the optimum number of neurons.

\section{Flow Curve Prediction by Using Artificial Neural Network Model}

In order to measure the performance of the developed ANN model, determination coefficient $\mathrm{R}^{2}$ and root mean square error (RMSE) measurements were used. The RMSE value for the estimated ANN model results is calculated according to Equation (1).

$$
R M S E=\sqrt{\frac{\left(y_{\text {exp }}-y_{\text {pre }}\right)^{2}}{N}}
$$

where $N$ is the total number of observations, $y_{\text {exp }}$ is experimental result, and $y_{\text {pre }}$ is predicted value. In order to verify the generalization capability of the developed ANN model, $25 \%$ of the data obtained by the experimental compression tests (flow curve data for $100{ }^{\circ} \mathrm{C}$ ) is reserved. By using the ANN model trained with the remaining $75 \%$ of the experimental data (flow curve data for $20,200,400{ }^{\circ} \mathrm{C}$ ), the material model predictions at $100{ }^{\circ} \mathrm{C}$ were obtained for different strain rate values. Comparison charts of predicted and target (real) data for strain rate 1, 10 and 50 values are given in Fig. 5,6,7, respectively. As seen in the figures, $R^{2}$ values are very high and the proposed ANN model predicted true stress values very close to the real data. Considering these graphs, it was observed that the difference between the predicted and actual true stress values is at most $14 \mathrm{MPa}$. As a result, it was concluded that the proposed ANN model reflected the input parameters very well and made promising predictions in very short CPU time as 8.10 seconds. 
Flow curve prediction of cold forging steel by artificial neural network model

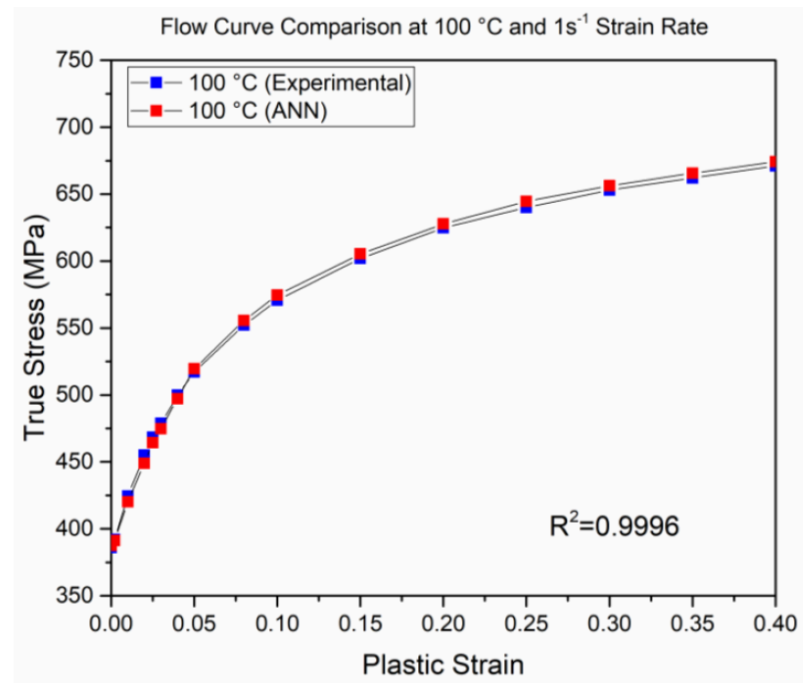

Fig. 5. Comparison of ANN predictions for the validation data set, strain rate: $1 \mathrm{~s}^{-1}$.

True stress values for strain rate of $1 \mathrm{~s}^{-1}$ at $100{ }^{\circ} \mathrm{C}$ was good agreement with the predicted values. The obtained true stress predictions had RMSE $=3.53$ and $\mathrm{R}^{2}=0.9996$ which confirm the generalization capability of ANN model.



Fig. 6. Comparison of ANN predictions for the validation data set, strain rate: $10 \mathrm{~s}^{-1}$.

True stress values for strain rate of $10 \mathrm{~s}^{-1}$ at $100{ }^{\circ} \mathrm{C}$ was also good agreement with the predicted values. The obtained true stress predictions had RMSE $=4.77$ and $\mathrm{R} 2=0.9996$ which also confirm the generalization capability of ANN model. 


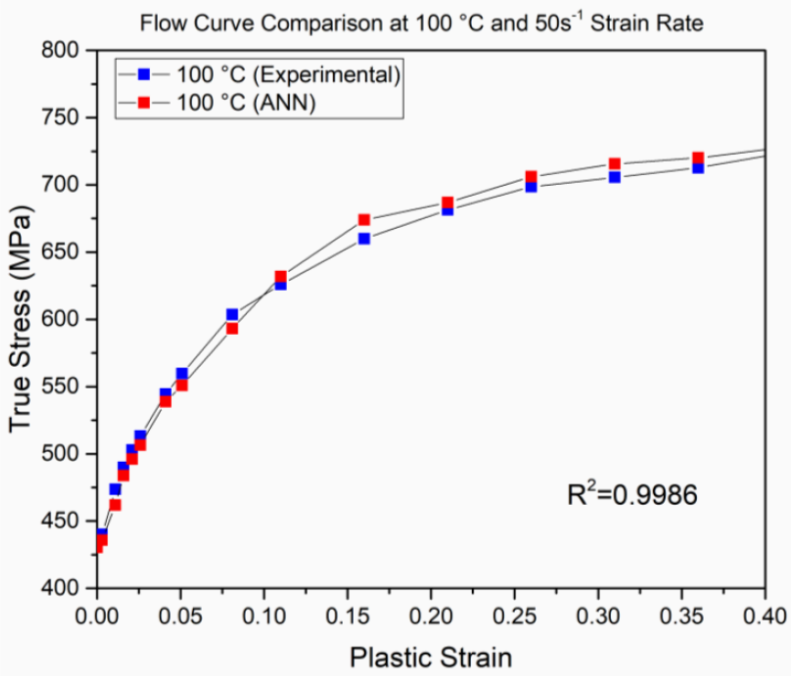

Fig. 7. Comparison of ANN predictions for the validation data set, strain rate: $50 \mathrm{~s}^{-1}$.

True stress values for strain rate of $50 \mathrm{~s}^{-1}$ at $100^{\circ} \mathrm{C}$ was also good agreement with the predicted values. The obtained true stress predictions had $\mathrm{RMSE}=7.31$ and $\mathrm{R}^{2}=0.9986$ which also confirm the generalization capability of ANN model.

\section{Conclusion}

A limited number of material models are preloaded on Simufact.forming software, so it finds values by interpolating or extrapolating for material property ranges that are not defined during analysis. Interpolating or extrapolating material properties causes the analysis results to be erroneous. For this reason, material models for the raw materials are obtained by experimental method and integrated into the finite element software. Although obtaining the material model with experimental methods and integrating it into the software improves the analysis results, it is very costly to create a material model experimentally for each temperature and strain rate value. Therefore, flow curves of a medium carbon alloy steel material, which is frequently used in bolt production by cold forming method, were experimentally obtained by compression tests within the scope of this study. Afterwards, ANN model was created to predict the flow curves of the material and the results were compared with the experimental data. Prediction results obtained with the ANN model were able to predict the material data allocated for verification at a high accuracy rate. As a result, it was concluded that the proposed ANN model reflected the input parameters very well and made promising predictions.

\section{Bibliography}

[1] Dhingra, A. K., and Rao, S. S. A Neural Network Based Approach To Mechanical Design Optimization. Engineering Optimization, 1992, 20(3), 187-203. DOI 10.1080/03052159208941280.

[2] JR, J. H. Garrett, Case, M. P., Hall, J. W., Yerramareddy, S., Herman, A., Sun, R., Ranjithan, S., and Westervelt, J. Engineering Applications of Neural Networks. Journal of Intelligent Manufacturing, 1993, 4, 1-21.

[3] Denizer, B. Artificial Neural Network Analysis of the Mechanical Properaties of Tungsten Fiber / Bulk Metallic Glass Matrix Composites via Neutron Diffraction and Finite Element Modelling. MS thesis, 2008, Iowa State University.

[4] Toros, S., and Ozturk, F. Flow Curve Prediction of Al-Mg Alloys under Warm Forming Conditions at Various Strain 
Flow curve prediction of cold forging steel by artificial neural network model

Rates by ANN. Applied Soft Computing, 2011, 11(2), 1891-1898. DOI 10.1016/j.asoc.2010.06.004.

[5] Pattanaik, L. N. Applications of Soft Computing Tools in Metal Forming: A State-of-Art Review. Journal of Machining \& Forming Technologies, 2013, 5(1/2), 1-15.

[6] Mehtedi, M. El, Forcellese, A., Greco, L., Pieralisi, M., and Simoncini, M. Flow Curve Prediction of ZAM100 Magnesium Alloy Sheets Using Artificial Neural Network-Based Models. Procedia CIRP, 2019, 79, 661-666. DOI 10.1016/ j.procir.2019.02.050.

[7] Merayo, D., Rodriguez-Prieto, A., and Camacho, A. M. Prediction of Physical and Mechanical Properties for Metallic Materials Selection Using Big Data and Artificial Neural Networks. IEEE Access, 2020, 8, 13444-13456. DOI 10.1109/ ACCESS.2020.2965769.

[8] Huynh, T.-C., Park, J.-H., Jung, H.-J., and Kim, J.-T. Quasi-Autonomous Bolt-Loosening Detection Method Using VisionBased Deep Learning and Image Processing. Automation in Construction, 2019, 105(May), 102844. DOI 10.1016/ j.autcon.2019.102844.

[9] Zhang, Y., Sun, X., Loh, K. J., Su, W., Xue, Z., and Zhao, X. Autonomous Bolt Loosening Detection Using Deep Learning. Structural Health Monitoring, 2020, 19(1), 105-122. DOI 10.1177/1475921719837509.

[10] ASTM E9-19. Standard Test Methods of Compression Testing of Metallic Materials at Room Temperature. ASTM International, 2019, West Conshohocken, PA.

[11] Haykin, S. Neural Networks; a Comprehensive Foundation. Prentice-Hall, 1999, Upper Saddle River.

[12] Lau, C. Neural Networks, Theoretical Foundations and Analysis. IEEE Press, 1992, New York, NY.

[13] McClelland, J. L., Rumelhart, D. E., and PDP Research Group. Parallel Distributed Processing. MIT Press, 1986, Cambridge, MA.

PDF automatically generated on 2021-05-20 08:22:33

Article url: https://popups.uliege.be/esaform21/index.php?id $=4140$

published by ULiège Library in Open Access under the terms and conditions of the CC-BY License

(https://creativecommons.org/licenses/by/4.0) 